06

\title{
Устойчивость полимерных композитов с оксидом вольфрама к воздействию электронного облучения
}

\author{
(C) Н.И. Черкашина
}

Белгородский государственный технологический университет им. В.Г. Шухова, 308012 Белгород, Россия

e-mail: cherkashina.ni@bstu.ru

Поступило в Редакцию 11 апреля 2019 г.

В окончательной редакции 11 апреля 2019 г.

Принято к публикации 27 мая 2019 г.

Представлены данные по физико-механическим и термическим свойствам полимерных композитов на основе полиимида и оксида вольфрама $\left(\mathrm{WO}_{3}\right)$. Расчетным и экспериментальным методами изучена устойчивость композитов к потоку быстрых электронов с энергией $0.5-5 \mathrm{MeV}$. В исследуемом энергетическом диапазоне представлены пробеги электронов в композитах с различным содержанием оксида вольфрама. Разница в экспериментальных и расчетных данных по пробегу электронов составила $\pm 15 \%$. Представлены результаты моделирования зависимости коэффициентов пропускания по числу частиц и энергии в зависимости от толщины композита оптимального состава, содержащего $60 \mathrm{wt} . \% \mathrm{WO}_{3}$, в случае падения электронов как перпендикулярно $(\varphi=0)$, так и при $\varphi=45^{\circ}$. Установлена возможность использования разработанного композита для защиты от электронного облучения в космическом пространстве.

Ключевые слова: полимерный композит, полиимид, комсмическое излучение, быстрые электроны, пробег электронов.

DOI: $10.21883 /$ JTF.2020.01.48671.163-19

\section{Введение}

Полимерные материалы активно применяются во многих отраслях промышленности: авиация, космические технологии, машиностроение, электроника и др. В космической технике переход к применению полимерных материалов взамен тяжелых металлических или керамометаллических обусловлен снижением веса конструкции, экономией топлива и тем самым снижению стоимости всей космической миссии $[1,2]$.

Однако применение чистых полимерных материалов в открытом космосе затруднено в силу комплекса факторов, влияющих на те или иные свойства полимера, основные из них следующие: глубокий вакуум, электроны и протоны радиационных поясов Земли, резкие перепады температур, микрометеоритные частицы и др. Все эти космические факторы снижают функциональные свойства материалов [3-5], что может привести к выводу из строя сам космический летательный объект.

В полимерных веществах проявляются самые разные радиационные эффекты при облучении, в том числе и в условиях космического пространства [6]. При облучении в полимерах возможно протекание двух типов реакции: поперечное сшивание или разрыв цепи. Процесс сшивания приводит к образованию химических связей между двумя смежными молекулами полимера. Эта реакция увеличивает молекулярную массу полимера, пока материал в конечном итоге не будет связан в нерастворимую трехмерную сеть. Формирование новых химических связей после облучения обычно приводит к необратимым последствиям. Как правило, они проявляются в изменении механических, диэлектрических и тепловых свойств. Разрыв цепи или разрушение молекул полимера уменьшает его молекулярный вес и увеличивает растворимость $[7,8]$. Так как большинство полимеров являются диэлектриками, то еще одним негативным последствием облучения электронного облучения в космическом пространстве является радиационное заряжение. Полимерные диэлектрики заряжаются падающим из космоса электронным потоком, и в них за счет энергии поглощенных электронов значительно увеличивается проводимость, а также необратимо изменяется внутренняя структура. Накопление большого электрического заряда в полимерах является отрицательным фактором, так как это может вызвать пробои в материале диэлектрика [9]. Электрические пробои в диэлектрике не только создают помехи, но и разрушают сами диэлектрики, ухудшая их эксплуатационные свойства. В результате пробоев в диэлектрике остаются следы в виде фигур Лихтенберга ветвистых деревьев разряда [10].

Введение различных наполнителей в полимер позволяет улучшить его механические и термические характеристики, делая материал более устойчивым к негативным факторам космического пространства. Кроме того, используя такие наполнители как $\mathrm{Bi}_{2} \mathrm{O}_{3}, \mathrm{Al}_{2} \mathrm{O}_{3}, \mathrm{PbO}$, можно повысить радиационно-защитные характеристики конечного композита [11-14].

Одним из перспективных наполнителей для повышения радиационно-защитных свойств полимеров является оксид вольфрама (VI). $\mathrm{WO}_{3}$ обладает высокой плотностью $7.2-7.4 \mathrm{~g} / \mathrm{cm}^{3}$, термической стойкостью, нетоксичен и имеет широкие технологические возможности 
получения [15-17]. Кроме того, $\mathrm{WO}_{3}$ обладает полупроводниковыми свойствами [18], что при введении его в диэлектрическую полимерную матрицу позволит значительно снизить или полностью исключить накопление большого электрического заряда при взаимодействии композита с быстрыми электронами в космическом пространстве. Большинство российских и зарубежных работ посвящено в основном изучению радиационнозащитных свойств композитов $\mathrm{c} \mathrm{WO}_{3}$ по отношению к рентгеновскому и гамма-излучению [19-22]. Работы по оценке радиационно-защитных свойств композитов c $\mathrm{WO}_{3}$ по отношению к электронному и протонному излучениям немногочисленны.

Наиболее перспективным полимерным связующим для создания композитов авиационно-космического назначения является полиимид, который обладает уникальным набором физических и механические свойств, таких как высокая износостойкость, стабильность в агрессивной среде, высокая ударная вязкость, широкий температурный диапазон эксплуатации $\left(-250\right.$ до $\left.+350^{\circ} \mathrm{C}\right)$ и др. [23]. В данной работе представлены расчетные и экспериментальные данные по прохождению пучка быстрых электронов через полимерные композиты на основе полиимидной матрицы и $\mathrm{WO}_{3}$ с оценкой их радиационно-защитных характеристик.

\section{1. Материалы и методы исследования}

В качестве связующего (матрицы) использовали термопластичный полиимид в виде прессовочного порошка марки ПИ-ПР-20. Молекулярная масса полиимида составляла $(100-120) \cdot 10^{3}$. Химическая структура используемого полиимида представлена на рис. 1 .

В качестве наполнителя использовали оксид вольфрама (VI) в виде порошка с размерами частиц до $2 \mu \mathrm{m}$.

Синтез композитов осуществлялся методом твердофазного компактирования хорошо гомогенизированной смеси связующего и наполнителя в определенных процентных соотношениях. Содержание наполнителя в композите варьировалось от 0 до $80 \mathrm{wt} \%$. Температура нагрева смеси составляла $350^{\circ} \mathrm{C}$, давление прессования не менее $200 \mathrm{MPa}$. Прессование композитов проводили с использованием автоматического гидравлического пресca Vaneox-40t automatic. Полученные композиты представляли собой таблетки диаметром $30 \mathrm{~mm}$ различной толщины.

Плотность композитов определяли методом гидростатического взвешивания.

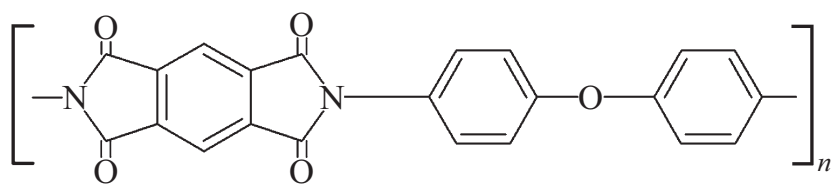

Рис. 1. Химическая структура используемого полиимида.
Таблица 1. Элементный химический состав разработанных композитов

\begin{tabular}{c|c|c|c|c|c}
\hline \multirow{2}{*}{$\begin{array}{c}\text { Содержание } \\
\mathrm{WO}_{3}, \%\end{array}$} & \multicolumn{5}{|c}{ Содержание атомов, \% } \\
\cline { 2 - 6 } & $\mathrm{C}$ & $\mathrm{N}$ & $\mathrm{O}$ & $\mathrm{H}$ & $\mathrm{W}$ \\
\hline & 68.11 & 7.57 & 21.62 & 2.70 & - \\
10 & 61.30 & 6.81 & 21.53 & 2.43 & 7.93 \\
20 & 54.49 & 6.05 & 21.44 & 2.16 & 15.86 \\
30 & 47.68 & 5.30 & 21.33 & 1.89 & 23.80 \\
40 & 40.86 & 4.55 & 21.25 & 1.62 & 31.72 \\
50 & 34.06 & 3.78 & 21.15 & 1.35 & 39.66 \\
60 & 27.24 & 3.03 & 21.06 & 1.08 & 47.59 \\
70 & 20.43 & 2.27 & 20.96 & 0.82 & 55.52 \\
80 & 13.62 & 1.51 & 20.87 & 0.54 & 63.46
\end{tabular}

Микротвердость композитов определяли по методу Виккерса с использованием микротвердомера Duramin-2 путем вдавливания четырехгранной алмазной пирамидки, которая имеет угол при вершине в $136^{\circ}$.

Механические испытания проводились на универсальной механической испытательной машине UTS-10.

Значение поверхностного сопротивления $(R)$ разработанных композитов определялось с помощью электродов, подсоединенных к электрометру. Удельное электрическое сопротивление $(\rho)$ вычисляли исходя из поверхностного сопротивления по формуле (1):

$$
\rho=\frac{R d b}{l}
$$

где $R$ - электрическое сопротивление, $d-$ толщина образца, $l$ - расстояние между электродами, $b$ размер композита (линейный).

Термостойкость композита оценивали путем определения температуры, при которой потеря массы достигала 5 wt.\% . Потерю массы регистрировали с использованием прибора синхронного термического анализа STA 449 F1 Jupiter (NETZSCH). Скорость нагрева $10^{\circ} \mathrm{C} / \mathrm{min}$. В качестве материала сравнения использовали оксид алюминия.

Для облучения разработанного композита электронами использовали „Установку для облучения электронным пучком диэлектрических материалов“, расположенную в лаборатории космического материаловедения в БГТУ им. В.Г. Шухова. Облучение образцов флюенсом электронов $5 \cdot 10^{15} \mathrm{~cm}^{-2}$ осуществлялось в вакууме при давлении не более $10^{-5} \mathrm{~Pa}$ (время облучения $72 \mathrm{~h}$, коэффициент ускорения 10, что соответствует $720 \mathrm{~h}$ облучения в космосе при интенсивности излучения $\left.2 \cdot 10^{7} \mathrm{~cm}^{-2} \cdot \mathrm{s}^{-1}\right)$. Диапазон энергии быстрых электронов, используемых для экспериментов, $0.5-5 \mathrm{MeV}$.

Для определения эффективного пробега электронов в полимерных композитах из них изготавливали таблетки различной толщины одного и того же химического состава. Композит помещали между счетчиком и источником электронного излучения. По мере увеличения 
Таблица 2. Основные физико-механические и термические характеристики разработанных композитов

\begin{tabular}{|c|c|c|c|c|c|c|c|c|c|}
\hline \multirow{2}{*}{ Параметр } & \multicolumn{9}{|c|}{ Содержание $\mathrm{WO}_{3}, \%$} \\
\hline & 0 & 10 & 20 & 30 & 40 & 50 & 60 & 70 & 80 \\
\hline Плотность, $\mathrm{g} / \mathrm{cm}$ & 1.43 & 1.61 & 1.77 & 1.99 & 2.23 & 2.57 & 3.05 & 3.46 & 4.35 \\
\hline Микротвердость, $\mathrm{HV}_{100}$ & 15.5 & 15.5 & 15.6 & 15.6 & 15.8 & 16.0 & 16.0 & 13.0 & 8.9 \\
\hline $\begin{array}{l}\text { Предел прочности } \\
\text { при растяжении, МРа }\end{array}$ & 77 & 89 & 103 & 119 & 132 & 145 & 157 & 121 & 63 \\
\hline $\begin{array}{l}\text { Удельное электрическое } \\
\text { сопротивление, } \Omega \cdot \mathrm{m}\end{array}$ & $10^{14}$ & $2 \cdot 10^{11}$ & $3 \cdot 10^{9}$ & $10^{7}$ & $2 \cdot 10^{5}$ & $4 \cdot 10^{4}$ & $8 \cdot 10^{3}$ & $2 \cdot 10^{3}$ & $10^{3}$ \\
\hline $\begin{array}{l}\text { Термостойкость (потеря массы } \\
\text { до } 5 \mathrm{wt} \%),{ }^{\circ} \mathrm{C}\end{array}$ & 458 & 461 & 463 & 466 & 469 & 472 & 475 & 478 & 481 \\
\hline
\end{tabular}

толщины используемого композита скорость счета частиц уменьшалась. За эффективный пробег принимали толщину композита, при которой интенсивность регистрируемого излучения была нулевая.

Для моделирования прохождения быстрых электронов через разработанный композит использовали стандартные формулы, представленные в [24]. Расчет производили исходя из элементного химического состава разработанных композитов (табл. 1).

\section{2. Результаты и их обсуждение}

\section{1. Исследование физико-механических свойств композитов}

Основные физико-механические и термические характеристики разработанных полимерных композитов в зависимости от содержания вводимого $\mathrm{WO}_{3}$ представлены в табл. 2.

Анализ полученных данных табл. 2 показал, что при введении предлагаемого наполнителя $\left(\mathrm{WO}_{3}\right)$ плотность композитов значительно возрастает. Это объясняется тем, что плотность исходного полиимида достаточно мала $\left(1.43 \mathrm{~g} / \mathrm{cm}^{3}\right)$, тогда как плотность $\mathrm{WO}_{3}$ значительно больше $-7.3 \mathrm{~g} / \mathrm{cm}^{3}$.

Наблюдается незначительное (3\%) увеличение микротвердости композита при введении до $60 \mathrm{wt} . \% \mathrm{WO}_{3}$, однако при большем содержании $\mathrm{WO}_{3}$, наблюдается снижение микротвердости (табл. 2). Аналогичная ситуации наблюдается с пределом прочности при растяжении он увеличивается с $77\left(0 \% \mathrm{WO}_{3}\right)$ до $157 \mathrm{MPa}(60 \mathrm{wt} . \%$ $\left.\mathrm{WO}_{3}\right)$, а при введении большего $60 \mathrm{wt} . \%$ наполнителя его значение резко падает до $63 \mathrm{MPa}(80 \mathrm{wt} . \%)$. Это объясняется тем, что при > $60 \mathrm{wt} . \% \mathrm{WO}_{3}$ в композите происходит нехватка связующего полиимида для связывания частиц $\mathrm{WO}_{3}$ в единый композит, что приводит к значительному снижению физико-механических свойств композита. Таким образом, 60 mass.\% - это максимальная степень наполнения полиимида данным наполнителем.
Значение удельного электрического сопротивления чистого полиимида очень высоко $-10^{14} \Omega \cdot \mathrm{m}$, материал является диэлектриком. Введение полупроводника $\mathrm{WO}_{3}$ в полиимид приводит к снижению удельного электрического сопротивления всего композита и создает ему полупроводниковые свойства (табл. 2).

Увеличение содержания наполнителя $\mathrm{WO}_{3}$ в композите незначительно, но все же увеличивает их термостойкость с $458^{\circ} \mathrm{C}\left(0 \mathrm{wt} . \% \mathrm{WO}_{3}\right)$ до $481^{\circ} \mathrm{C}\left(80 \mathrm{wt} . \% \mathrm{WO}_{3}\right)$ (табл. 2).

\section{2. Исследование пробега электронов в композитах}

Расчетным методом было проведено исследование прохождения быстрых электронов с энергией 0.5-5 MeV в разработанных полимерных композитах. Для расчетов использовали известные физикоматематические формулы [24]. Так как разработанный полимерный композит является гетерогенным веществом, т.е. состоит из атомов нескольких химических элементов, то для определения тормозной способности дополнительно применяли правило Брэгга (композиционный закон Брэгга):

$$
\left(-\frac{d E}{\rho d x}\right)=\sum_{i} \frac{\rho_{i}}{\rho}\left(-\frac{d E}{\rho d x}\right)_{i},
$$

где $\rho_{i}$ и $(-d E / \rho d x)_{i}$ - плотность и тормозная способность вещества из $i$-элемента.

Известно, что потери энергии движущимися электронами в веществе подразделяются на ионизационные и на радиационные. Энергетическая зависимость удельных ионизационных потерь падает с увеличением скорости до кинетических энергий, равных удвоенной энергии покоя электрона, а затем медленно поднимается. Радиационные потери наблюдаются при ускоренном движении свободной заряженной частицы в электрическом поле ядра. Для расчетов ионизационных потерь $(-d E / d x)_{c o l}$ 
использовали формулу

$$
\begin{aligned}
& \left(-\frac{d E}{d x}\right)_{c o l}=\left(\rho_{W} \frac{Z_{W}}{A_{W}} F\left(E_{k}, I_{W}\right)+\rho_{O} \frac{Z_{O}}{A_{O}} F\left(E_{k}, I_{O}\right)\right. \\
& \left.+\rho_{C} \frac{Z_{C}}{A_{C}} F\left(E_{k}, I_{C}\right)+\rho_{N} \frac{Z_{N}}{A_{N}} F\left(E_{k}, I_{N}\right)+\rho_{H} \frac{Z_{H}}{A_{H}}\right) F\left(E_{k}, I_{H}\right),
\end{aligned}
$$

где

$$
\begin{aligned}
& F\left(E_{k}, I\right)=\frac{K}{2 \beta^{2}}\left[\ln \left(\frac{m_{e} c^{2} E_{k}}{I^{2}} \frac{\beta^{2}}{2\left(1-\beta^{2}\right)}\right)\right. \\
& -\left(2 \sqrt{1-\beta^{2}}-1+\beta^{2}\right) \ln 2+1-\beta^{2} \\
& \left.+\frac{1}{8}\left(1-\sqrt{1-\beta^{2}}\right)^{2}\right]
\end{aligned}
$$

где $\quad K=0.307 \mathrm{MeV} /\left(\mathrm{g} / \mathrm{cm}^{2}\right), \quad m_{e} c^{2}=0.511 \mathrm{MeV}$, $r_{e}=2.8 \cdot 10^{-13} \mathrm{~cm}, N_{A}-$ число Авогадро, $\rho-$ плотность вещества, $I-$ средний ионизационный потенциал атома вещества среды, $\beta=\sqrt{1-\frac{\left(m_{e} c^{2}\right)^{2}}{\left(m_{e} c^{2}+E_{k}\right)^{2}}}$.

Радиационные потери $(-d E / d x)_{\mathrm{rad}}$ рассчитывали по формуле

$$
\begin{aligned}
\left(-\frac{d E}{d x}\right)_{\text {rad }} & =\left(\rho_{W} \frac{Z_{W}^{2}}{A_{W}}+\rho_{O} \frac{Z_{O}^{2}}{A_{O}}+\rho_{C} \frac{Z_{C}^{2}}{A_{C}}\right. \\
& \left.+\rho_{N} \frac{Z_{N}^{2}}{A_{N}}+\rho_{H} \frac{Z_{H}^{2}}{A_{H}}\right) G\left(E_{k}\right),
\end{aligned}
$$

где

$$
\begin{aligned}
G\left(E_{k}\right) & =\frac{K \alpha}{4 \pi} \frac{\varepsilon}{m}\left[\frac{12 \varepsilon^{2}+4 m_{e}^{2} c^{4}}{3 \varepsilon p} \ln \left(\frac{\varepsilon+p}{m_{e} c^{2}}\right)\right. \\
& -\frac{(8 \varepsilon+6 p) m^{2} c^{4}}{3 \varepsilon p^{2}}\left(\ln \left(\frac{\varepsilon p}{m_{e} c^{2}}\right)\right)^{2}-\frac{4}{3} \\
& \left.+\frac{2 m^{2} c^{4}}{\varepsilon p} F\left(\frac{2 p(\varepsilon+p)}{m^{2} c^{4}}\right)\right],
\end{aligned}
$$

где $\varepsilon=E_{K}+m_{e} c^{2}-$ полная энергия электрона; $\alpha=1 / 137$ - постоянная тонкой структуры; $p-$ импульс электрона.

На рис. 2 представлены графики зависимости ионизационных (рис. 2,a) и радиационных (рис. 2,b) потерь быстрых электронов от их начальной энергии для композитов различных составов. Удельные суммарные потери электронов в композите определяются суммой ионизационных и радиационных потерь.

Анализ данных, представленных на рис. 2, показал, что при возрастании содержания $\mathrm{WO}_{3}$ в композите значительно увеличиваются как ионизационные, так и радиационные потери быстрых электронов в композитах. Наименьшие потери электронов наблюдаются у материала без наполнителя, а наибольшие соответственно у композита c 80 mass.\% $\mathrm{WO}_{3}$. Для одного и того же состава при большей исходной энергии электронов
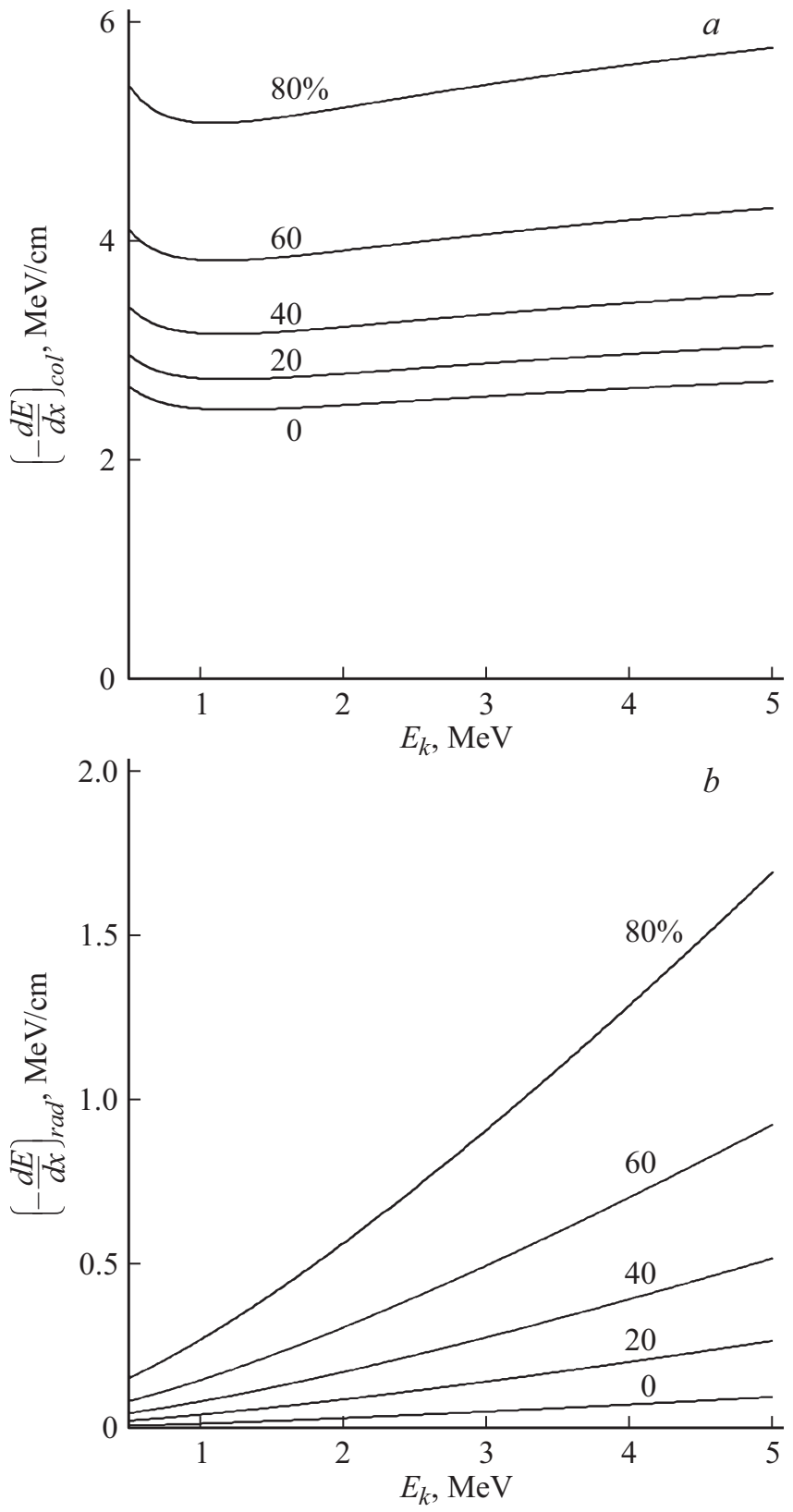

Рис. 2. Удельные ионизационные $(a)$ и радиационные $(b)$ потери быстрых электронов в полимерном композите при различном содержании $\mathrm{WO}_{3}$.

наблюдается увеличение и ионизационных, и радиационных потерь быстрых электронов в композите. Анализ кривых на рис. 2 показывает, что наибольшие потери при прохождении электронов через предлагаемые композиты обусловлены ионизационными потерями, тогда как радиационные потери в разы меньше. Если рассматривать отдельный вклад каждого элемента в удельные потери электронов при прохождении через композит, то можно отметить, что наибольший вклад вносит атом W, затем атомы С и О. Вклад атомов $\mathrm{N}$ и $\mathrm{H}$ незначителен.

При столкновении с атомными электронами и ядрами быстрые электроны значительно отклоняются от 


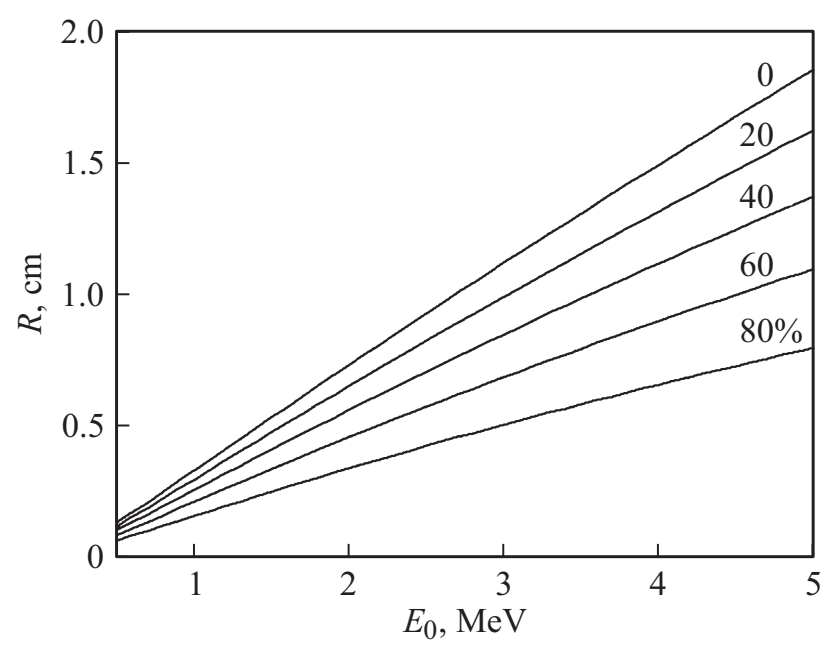

Рис. 3. Зависимость среднего пробега электрона в полимерном композите от его начальной кинетической энергии при различных содержаниях $\mathrm{WO}_{3}$.

первоначального направления движения и двигаются по извилистой траектории. Поэтому для электронов практический интерес представляет не истинный линейный пробег, а эффективный, определяемый минимальной толщиной материала, измеряемой в направлении исходной скорости пучка и соответствующей полному поглощению электронов. Он равен толщине вещества, которое поглощает электроны. На основании данных по удельным ионизационным и радиационным потерям быстрых электронов были рассчитаны эффективные пробеги электронов различной энергии в разработанных композитах по формуле:

$$
R\left(E_{0}\right)=\int_{0}^{E_{0}} \frac{d E_{k}}{\left(-\frac{d E}{d x}\right)} .
$$

На рис. 3 представлены графики зависимости среднего пробега электрона в полимерном композите от его начальной кинетической энергии при различных содержаниях $\mathrm{WO}_{3}$.

Как и ожидалось, с увеличением содержания $\mathrm{WO}_{3}$ пробег электронов (при одинаковой первоначальной энергии) уменьшается, что соответствует большим потерям энергии в композите. Для композитов одинакового состава при увеличении первоначальной энергии электронов пробег электронов увеличивается. По данным рис. 3 можно утверждать, что эффективный пробег электронов в разработанных высоконаполненных (содержание $\left.\mathrm{WO}_{3}>60 \mathrm{wt} . \%\right)$ композитах достаточно мал во всем рассматриваемом диапазоне начальных энергий электрона (от 0.5 до $5 \mathrm{MeV}$ ), что говорит о перспективе его использования для защиты от воздействия электронов в космическом пространстве.

Для оценки правильности полученных расчетным путем данных по эффективному пробегу электронов про-
Таблица 3. Экспериментальные значения эффективного пробега электронов

\begin{tabular}{c|l|l|l|l|l}
\hline \multirow{2}{*}{$\begin{array}{c}\text { Энергия } \\
\text { электронов, }\end{array}$} & \multicolumn{5}{|c}{ Эффективный пробег, cm } \\
\cline { 2 - 6 } $\mathrm{MeV}$ & - & 20 & 40 & 60 & 80 \\
\cline { 2 - 6 } & 0.15 & 0.15 & 0.15 & 0.1 & 0.1 \\
\hline 0.5 & 0.35 & 0.3 & 0.25 & 0.25 & 0.2 \\
1 & 0.75 & 0.65 & 0.55 & 0.45 & 0.35 \\
2 & 1.15 & 1 & 0.85 & 0.7 & 0.5 \\
3 & 1.5 & 1.35 & 1.05 & 0.9 & 0.65 \\
4 & 1.9 & 1.65 & 1.4 & 1.1 & 0.8 \\
5 & & & &
\end{tabular}

ведены экспериментальные исследования эффективных пробегов в разработанных композитах. В табл. 3 представлены экспериментальные данные по полученному эффективному пробегу с использованием „Установки для облучения электронным пучком диэлектрических материалов“, расположенной в лаборатории космического материаловедения в БГТУ им. В.Г. Шухова.

Анализ полученных экспериментальных (табл. 3) и расчетных (рис. 3) результатов по среднему пробегу электронов в разработанном полимерном композите показал большую сходимость полученных данных. Разница в экспериментальных и расчетных данных по пробегу электронов составляет $\pm 15 \%$. Такое отклонение может быть вызвано, во-первых, погрешностью детектора, который производит регистрацию и подсчет импульсов. А во-вторых, геометрическими особенностями синтезированных композитов. Для определения эффективного пробега электронов в полимерных композитах из них изготавливали таблетки различной толщины одного и того же химического состава. Толщина образцов составляла от 0.1 до $2 \mathrm{~cm} \mathrm{с} \mathrm{шагом} 0.05 \mathrm{~cm}$. Сделать меньший шаг для толщины композитов не представлялось технически возможным. Поэтому в табл. 3 можно наблюдать, что значение эффективного пробега электронов при $E=0.5 \mathrm{MeV}$ в композитах с 60 и $80 \mathrm{wt} . \% \mathrm{WO}_{3}$ одинаково и составляет $0.1 \mathrm{~cm}$. Вероятнее всего, эффективный пробег для электронов при $E=0.5 \mathrm{MeV}$ в композите c $80 \mathrm{wt} . \% \mathrm{WO}_{3}$ меньше, чем в композите с $60 \mathrm{wt} . \% \mathrm{WO}_{3}$.

\section{3. Исследование коэффициентов пропускания электронов в композитах}

При расчете эффективного пробега электронов и проведения экспериментальных исследований учитывалось то, что пучок электронов проходил перпендикулярно (под прямым углом) к поверхности композитов. Однако в реальных условиях космического пространства электроны будут проходить через материал (различные элементы космического аппарата) под разными углами. Поэтому представляется интерес по изучению прохождения электронов под различными углами $\varphi$ (угол 


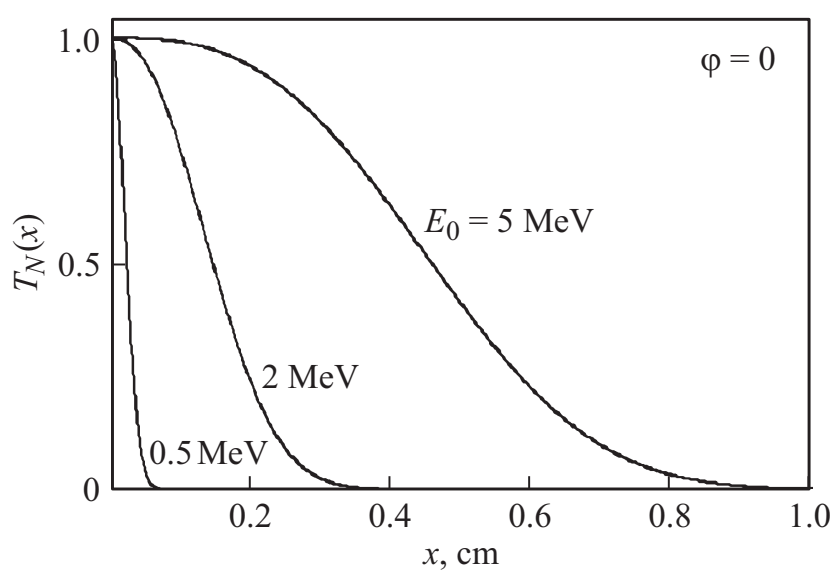

Pис. 4. Коэффициент пропускания числа электронов при нормальном падении на композит для разных начальных энергий электрона.

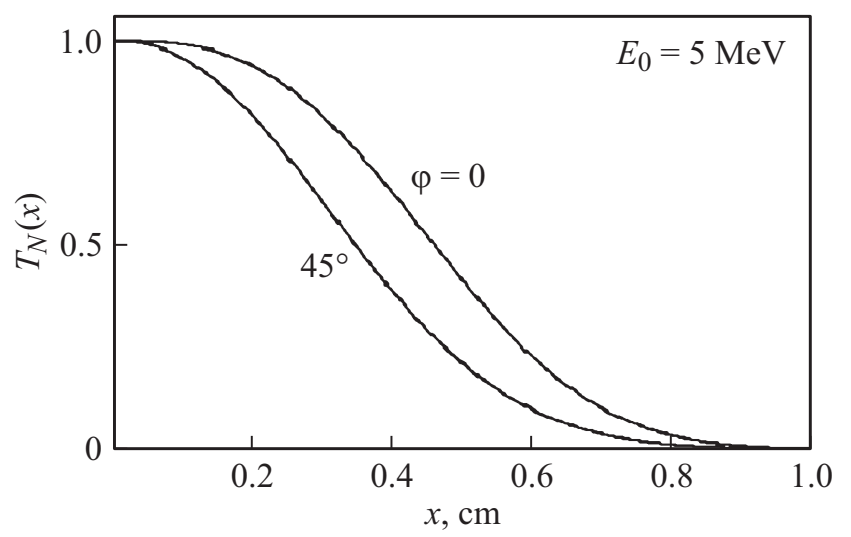

Рис. 5. Коэффициент пропускания числа электронов при разных углах падения с начальной энергией электронов $5 \mathrm{MeV}$.

между нормалью к поверхности композита и путем электрона) и анализ коэффициентов пропускания через прошедший композит.

Для расчета коэффициентов пропускания по числу частиц электронов использовали следующую формулу:

$$
T_{N}(x)=\exp \left[-\beta\left(\frac{x}{R_{e x}}\right)^{\alpha}\right],
$$

где

$$
\begin{aligned}
& R_{e x}\left(E_{0}, Z, \phi\right)=\cos ^{2} \frac{\phi}{2}\left(\frac{107.2-Z}{5.442 Z-1312}\right. \\
& \left.+\frac{292.7-Z}{4.163 Z+561.3} E_{0}+\frac{Z-2.797}{83.86 Z+587.5} E_{0}^{2}\right) \frac{1}{\rho},
\end{aligned}
$$

где $R_{e x}$ - экстраполированный пробег электронов.

$$
\begin{aligned}
\alpha & =1+\frac{5.5-0.1\left(3.4-E_{0}\right)^{2}}{0.398-0.032 E_{0}}(\cos \phi-0.1564) \\
& +0.0125\left(E_{0}-2\right)(50-Z)(\cos \phi-0.1564)^{3}
\end{aligned}
$$

для $Z<50, E>2 \mathrm{MeV}$,

$$
\alpha=1+\frac{5.5-0.1\left(3.4-E_{0}\right)^{2}}{Z^{0.398-0.032 E_{0}}}(\cos \varphi-0.1564)
$$

- в остальных случаях,

$$
\beta=2.59-0.0076(Z-6) \text {. }
$$

Коэффициент пропускания по энергии имеет следующий вид:

$$
\begin{gathered}
T_{E}(x)=\exp \left[-\beta_{E}\left(\frac{x}{R_{e x}}\right)^{\alpha_{E}}\right], \\
\alpha_{E}=0.78+\frac{Z+24}{0.93 Z+13.7}(\cos \varphi-0.1564), \\
\beta_{E}=\frac{Z+32.6}{0.524 Z+10.8} .
\end{gathered}
$$

Так как по данным физико-механических характеристик наилучшими свойствами обладает композит, содержащий $60 \mathrm{wt} . \% \mathrm{WO}_{3}$, то расчет коэффициентов пропускания представлен именно для этого состава. На

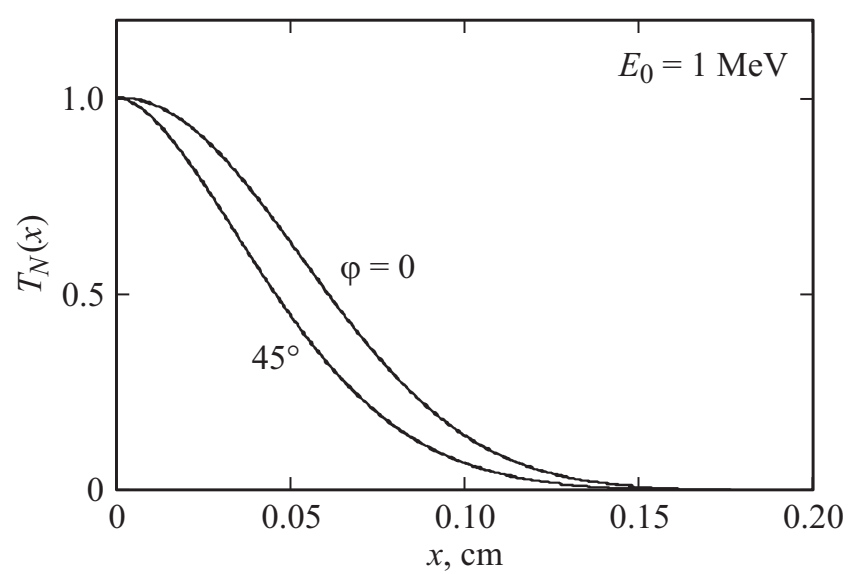

Рис. 6. Коэффициент пропускания числа электронов при разных углах падения с начальной энергией электронов $1 \mathrm{MeV}$.

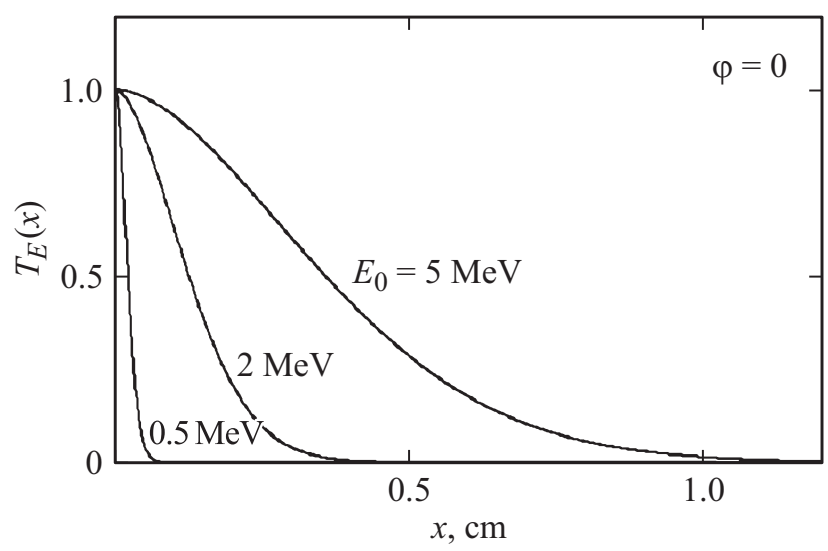

Рис. 7. Энергетический коэффициент пропускания при нормальном падении электронов на композит для разных начальных энергий электрона. 
рис. 4-7 графически представлены результаты моделирования зависимости коэффициентов пропускания по числу частиц и энергии в зависимости от толщины исследуемого композита, содержащего $60 \mathrm{wt} . \% \quad \mathrm{WO}_{3}$, для углов и начальных энергий, представленных на рисунках. Анализ полученных данных, представленных на рис. 4-7, говорит о высокой стойкости исследуемого полиимидного композита с оксидом вольфрама в качестве наполнителя по отношению к потоку быстрых электронов в случае их падения как перпендикулярно $(\varphi=0)$, так и при $\varphi=45^{\circ}$ в различном энергетическом диапазоне исследований $(E=0.5-5 \mathrm{MeV})$.

\section{Заключение}

В работе рассмотрено воздействие быстрых электронов с энергией от 0.5 до $5 \mathrm{MeV}$ на полиимидный композит, наполненный оксидом вольфрама теоретическим и экспериментальным способами.

По расчетным данным установлено, что при возрастании содержания оксида вольфрама в композите значительно увеличиваются как ионизационные, так и радиационные потери быстрых электронов. Наибольшие потери при прохождении электронов через предлагаемые композиты обусловлены ионизационными потерями, тогда как радиационные потери в разы меньше. Если рассматривать отдельный вклад каждого элемента в удельные потери электронов при прохождении через композит, то наибольший вклад вносит атом W, затем атомы $\mathrm{C}$ и $\mathrm{O}$. Вклад атомов $\mathrm{N}$ и Н незначителен.

Экспериментальные данные по эффективному пробегу электронов получены с использованием „Установки для облучения электронным пучком диэлектрических материалов“, расположенной в лаборатории космического материаловедения в БГТУ им. В.Г. Шухова. Установлена большая сходимость данных экспериментальных и расчетных результатов по среднему пробегу электронов в разработанных полимерных композитах. Разница в экспериментальных и расчетных данных по пробегу электронов составляет $\pm 15 \%$.

Установлена высокая стойкость полимерного композита по отношению к потоку быстрых электронов в случае их падения как перпендикулярно $(\varphi=0)$, так и при $\varphi=45^{\circ}$, оцениваемая по коэффициенту пропускания электронов через композит.

\section{Финансирование работы}

Работа выполнена в рамках гранта Российского научного фонда (проект № 19-19-00316).

\section{Конфликт интересов}

Авторы заявляют, что у них нет конфликта интересов.

\section{Список литературы}

[1] Власенко А.В., Скрябин В.В. // Актуальные проблемы авиации и космонавтики. 2016. Т. 1. С. 71-73.

[2] Fielding L.A., Hillier J.K., Burchell M.J., Armes S.P. // Chem. Commun. 2015. N 51. P. 16886-16899.

DOI: $10.1039 / \mathrm{c} 5 \mathrm{cc} 07405 \mathrm{c}$

[3] Шувалов В.А., Токмак Н.А., Письменный Н.И., Кочубей Г.С. // Космічна наука і технологія. 2012. Т. 18. № 3. C. 10-19.

[4] Черкашина Н.И., Павленко А.В. // ЖТФ. 2018. Т. 88. Вып. 4. C. 587-591. DOI: 10.21883/JTF.2018.04.45728.2237

[5] Li C., Mikhailov M.M., Neshchimenko V.V. // Nucl. Instrum. Meth. B. 2014. Vol. 319. P. 123-127. DOI: 10.1016/j.nimb.2013.11.007

[6] Радиационные эффекты в космосе. Ч. 2. Воздействие космической радиации на электротехнические материалы / И.П. Безродных, А.П. Тютнев, В.Т. Семёнов. М.: АО „Корпорация „ВНИИЭМ“, 2016. 122 с.

[7] Kacarevic-Popovic Z., Kostosk I.D., Novakovic L., Miljevic N., Secerov B.J. // Serb. Chem. Soc. 2004. Vol. 69. P. 1029-1041.

[8] Faltermeier A., Reicheneder C., Römer P., Castro-Laza A., Proff P. // J. Orofac. Orthop. 2014. Vol. 75. N 5. P. 334-344. DOI: $10.1007 / \mathrm{s} 00056-014-0229-5$

[9] Алексеев Г.С., Гришин А.В., Горностай-Польский С.А., Грунин А.В., Данилова И.М., Куимова Н.А., Лазарев С.А., Молитвин А.М., Тихонов А.И., Ткачук Д.В. // VIII Харитоновские чтения. Саров: РФЯЦ-ВНИИЭФ, 2007. С. 11-19.

[10] Garrett H., Whittlesey A. Guide to Mitigating Spacecraft Charging Effects, Wiley, 2012. 178 p.

[11] Gavrish V.M., Baranov G.A., Chayka T.V., Derbasova N.M., Lvov A.V., Matsuk Y.M. // IOP Conf. Ser.: Mater. Sci. Eng. 2016. Vol. 110. P. 012028. DOI: $10.1088 / 1757-899 \mathrm{X} / 110 / 1 / 012028$

[12] Osman A.M., Abdel-Monem A.M., Mansour Aly F.F. // J. Chem., Biolog. Phys. Sci. 2016. Vol. 6. P. 302-315.

[13] Aycik G.A., Belgin E.E. // Intern. J. Chem. Chem. Engineer. Systems. 2018. Vol. 3. P. 1-4.

[14] Павленко В.И., Ястребинский Р.Н., Едаменко О.Д., Ястребинская А.В. // Вестник Белгородского гос. технол. унта им. В.Г. Шухова. 2009. № 3. С. 62-66.

[15] Шаповалов В.И., Лапшин А.Е., Комлев А.Е., Арсентьев М.Ю., Комлев А.А. // ЖТФ. 2013. Т. 83. Вып. 1. C. $73-83$.

[16] Fakhri A., Behrouz S. // Solar Energy. 2015. Vol. 112. P. 163-168. DOI: $10.1016 /$ j.solener.2014.11.014

[17] Zou B.X., Wang Y., Huang F. // Advanc. Mater. Res. 2014. Vol. 968. P. 72-75.

DOI: 10.4028/www.scientific.net/AMR.968.72

[18] Shapovalov V.I., Komlev A.E., Komlev A.A., Morozova A.A., Lapshin A.E. // Glass Phys. Chem. 2013. Vol. 39. N 6. P. 664-666. DOI: 10.1134/S1087659613060096

[19] Tekin H.O., Singh V.P., Manici T. // Appl. Radiat. Isotopes. 2017. Vol. 121. P. 122-125.

DOI: 10.1016/j.apradiso.2016.12.040

[20] Malekie S., Hajiloo N. // Chin. Phys. Lett. 2017. Vol. 34. N 10. P. 108102. DOI: 10.1088/0256-307X/34/10/108102

[21] Azman N.Z., Siddiqui S.A., Low I.M. // Mater. Sci. Eng. C. Mater. Biol. Appl. 2013. Vol. 33. N 8. P. 4952-7. DOI: $10.1016 /$ j.msec.2013.08.023 
[22] Ali K.M., Mohammad K.K., Atallah F.S. // J. Rad. Nucl. Applicat. 2018. N 3. P. 191-197. DOI: $10.18576 / \mathrm{jrna} / 030309$

[23] Yoo K.-P., Lee M.J., Kwon K.-H., Jeong J., Min N.-K. // Thin Solid Films. 2010. Vol. 518. P. 5986-5991.

DOI: $10.1016 /$ j.tsf.2010.05.099

[24] Cherkashina N.I., Pavlenko V.I., Noskov A.V. /I Radia. Phys. Chem. 2019. Vol. 159. P. 111-117. DOI: $10.1016 /$ j.radphyschem.2019.02.041 